\title{
Symmetries in the Gross-Neveu Phase Diagram
}

\author{
Gerald Dunne
}

University of Connecticut

crystalline phases of GN models: gap equation and integrable hierarchies

G.Başar \&GD, arxiv:0803.1501, PRL 100, 200404 (2008) arxiv:0806.2659, PRD 78, 065022 (2008)

G.Başar, GD \& M.Thies, arxiv: 0903.1868, PRD in press F.Correa, GD \& M.Plyushchay, arxiv: 0904.2768

energy-reflection symmetry of periodic QES systems

GD \& M.Shifman, hep-th/0204224, Ann. Phys. 299, 143 (2002) 


\section{Gross-Neveu Models}

$\mathrm{GN}_{2} \quad \mathcal{L}_{\mathrm{GN}}=\bar{\psi} i \not \partial \psi+\frac{g^{2}}{2}(\bar{\psi} \psi)^{2}$

$\chi \mathrm{GN}_{2}$

$\mathrm{NJL}_{2}$
Gross/Neveu, 1974

$$
\psi \rightarrow \gamma^{5} \psi
$$

$\psi \rightarrow e^{i \alpha \gamma^{5}} \psi$

- renormalizable; large $\mathrm{N}_{\mathrm{f}}$ limit

- asymptotically free

- chiral symmetry breaking

- dynamical mass generation

- self-bound baryonic states $m_{B}=\frac{2}{\pi} m$ 


\section{lattice $\mathrm{GN}_{2}$ model}

de Forcrand/Wenger 2006

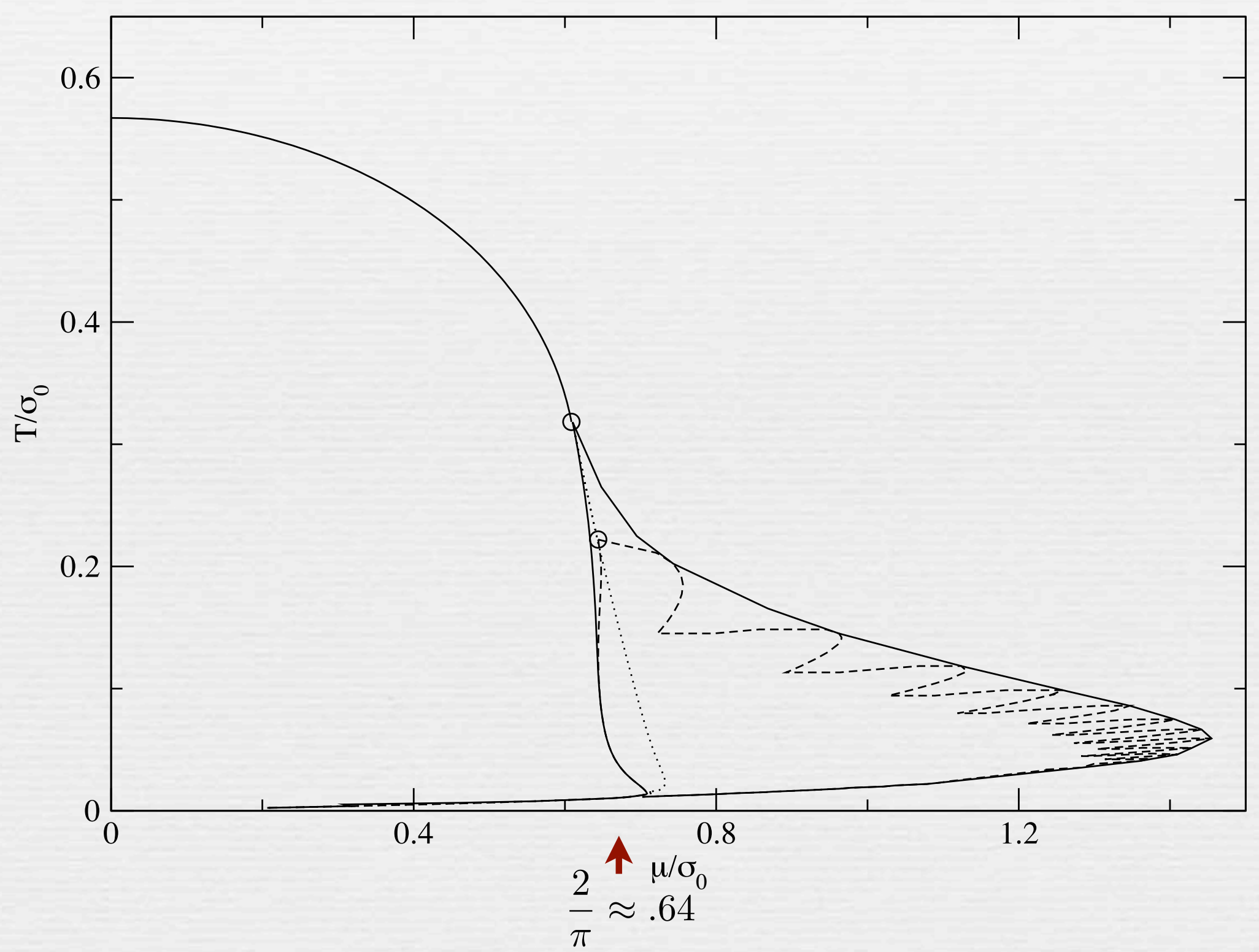




\section{Condensed matter analogues}

trans-polyacetylene $=\mathrm{GN}_{2}$

Su, Schreiffer, Heeger, 1979

dimerization = discrete chiral symmetry of GN model polaron crystal Brazovskii, 1980; Horovitz, 1981

1 dim. Peierls-Fröhlich electron-phonon model

Mertsching/Fischbeck, 1981; Belokolos et al, 1981

inhomogeneous superconductors and ferromagnetism

Machida/Nakanishi, 1984

magnetic field $=\mu$

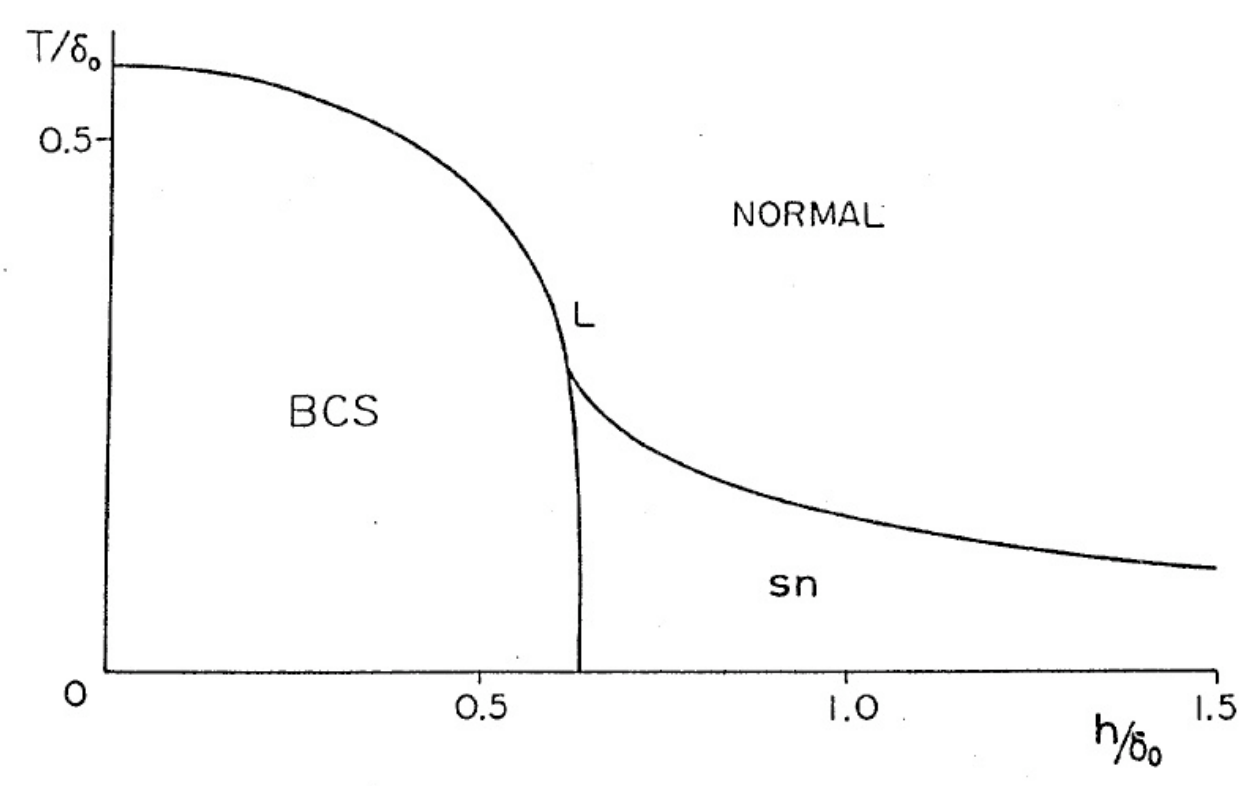


inhomogeneous gap equation : $\mathrm{GN}_{2}$

$$
\frac{\Sigma(x)}{g^{2} N}=\frac{\delta}{\delta \Sigma(x)} \ln \operatorname{det}[\not \partial+\Sigma(x)]
$$

DHN(1975): inverse scattering

$\Rightarrow$ reflectionless potentials: $\quad V_{ \pm}=\Sigma^{2} \pm \Sigma^{\prime}$

single kink: $\quad \Sigma(x)=m \tanh (m x)$

Thies/Urlichs (2005): finite-gap potentials: $V_{ \pm}=\Sigma^{2} \pm \Sigma^{\prime}$

kink crystal: $\quad \Sigma(x)=m \nu \frac{\operatorname{sn}(m x ; \nu) \operatorname{cn}(m x ; \nu)}{\operatorname{dn}(m x ; \nu)}$ 
kink crystal $\quad \Sigma(x)=m \nu \frac{\operatorname{sn}(m x ; \nu) \operatorname{cn}(m x ; \nu)}{\operatorname{dn}(m x ; \nu)}$

$$
\nu=0.999
$$

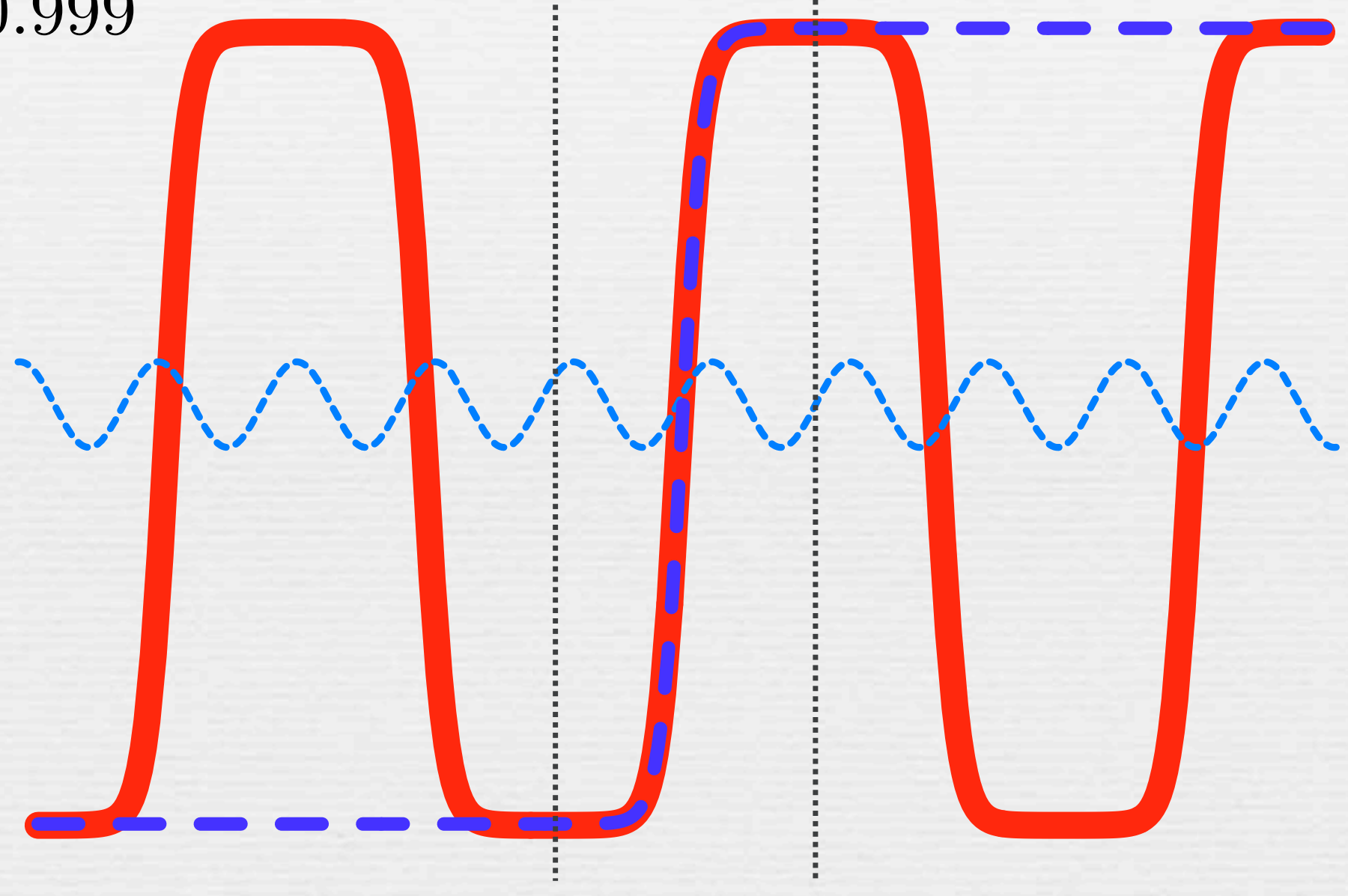


complex gap equation: $\mathrm{NJL}_{2} \quad \Delta=\Sigma-i \Pi$

$$
\frac{\Delta(x)}{g^{2} N}=\frac{\delta}{\delta \Delta^{*}(x)} \ln \operatorname{det}\left[\not \partial+\left(\Sigma(x)-i \gamma^{5} \Pi(x)\right)\right]
$$

Shei (1976): inv. scattering: reflectionless Dirac system

$$
\Delta(x)=m \frac{\cosh \left(m \sin \left(\frac{\theta}{2}\right) x-i \frac{\theta}{2}\right)}{\cosh \left(m \sin \left(\frac{\theta}{2}\right) x\right)}
$$

twisted kink

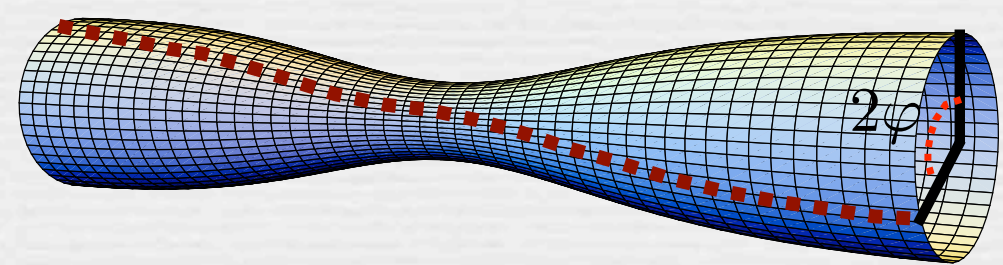

GD \& Basar (2008): finite-gap Dirac system

$$
\Delta(x)=A \frac{\sigma\left(A x+i \mathbf{K}^{\prime}-i \frac{\theta}{2}\right)}{\sigma\left(A x+i \mathbf{K}^{\prime}\right) \sigma\left(i \frac{\theta}{2}\right)} e^{i Q x}
$$


twisted kink crystal: general solution of $\mathrm{NJL}_{2}$ gap equation

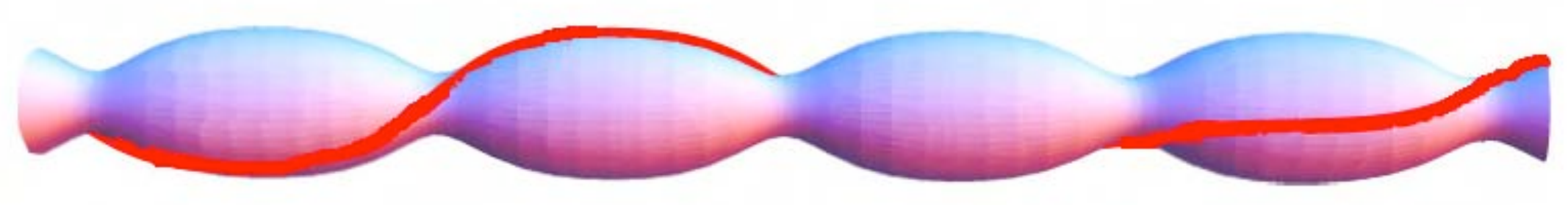

real kink crystal

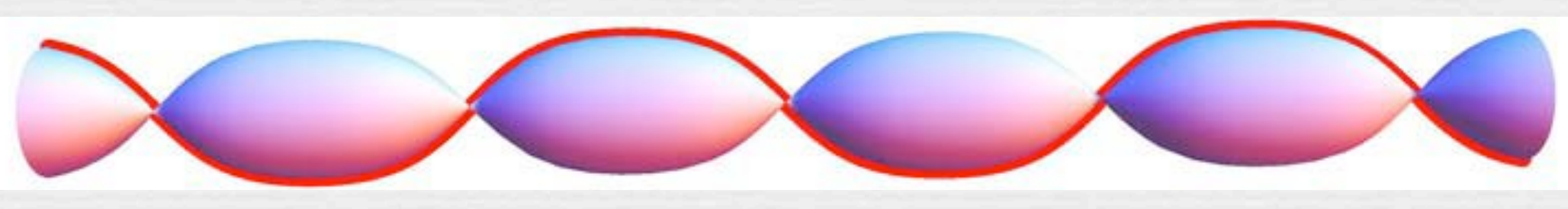

spiral crystal

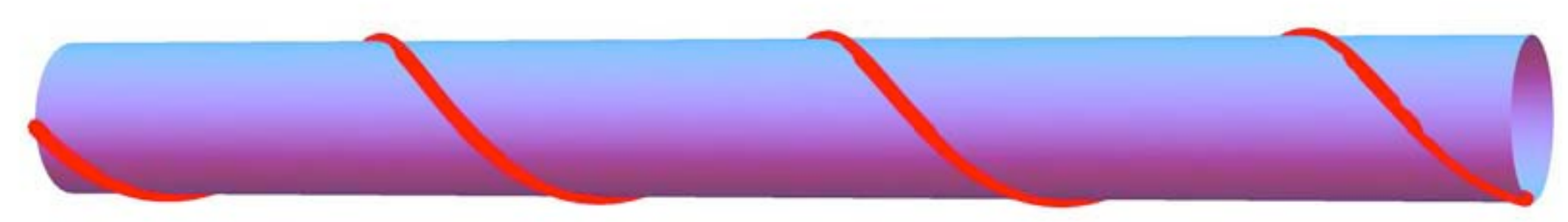




\section{single-particle spectra}

$$
E_{4}=q+\lambda
$$

$E_{4}$

$E_{4}$

$$
q
$$

$E_{3}$

$$
q=0
$$$$
E_{2}
$$

$E_{1}=q-\lambda$

$E_{1}$

$$
\Delta(x)=A \frac{\sigma\left(A x+i \mathbf{K}^{\prime}-i \frac{\theta}{2}\right)}{\sigma\left(A x+i \mathbf{K}^{\prime}\right) \sigma\left(i \frac{\theta}{2}\right)} e^{i Q x}
$$




\section{solving the (complex) gap equation}

$$
\begin{gathered}
\frac{\Delta(x)}{g^{2} N}=\frac{\delta}{\delta \Delta^{*}(x)} \ln \operatorname{det}\left[\not \partial+\left(\Sigma(x)-i \gamma^{5} \Pi(x)\right)\right] \\
H=-i \gamma^{5} \frac{d}{d x}+\gamma^{0} \Sigma(x)+i \gamma^{1} \Pi(x)=\left(\begin{array}{cc}
-i \frac{d}{d x} & \Delta(x) \\
\Delta^{*}(x) & i \frac{d}{d x}
\end{array}\right)
\end{gathered}
$$

Bogoliubov/de Gennes hamiltonian

resolvent: Gorkov Green's function $\quad R(x ; E) \equiv\left\langle x\left|\frac{1}{H-E}\right| x\right\rangle$ spectral function $\quad \rho(E)=\frac{1}{\pi} \operatorname{Im} \int d x \operatorname{tr} R(x ; E+i \epsilon)$

Eilenberger eqn: $\frac{\partial}{\partial x} R(x ; E) \sigma_{3}=i\left[\left(\begin{array}{cc}E & -\Delta(x) \\ \Delta^{*}(x) & -E\end{array}\right), R(x ; E) \sigma_{3}\right]$ 


\section{solving the (complex) gap equation}

$$
\frac{\Delta(x)}{g^{2} N}=\frac{\delta}{\delta \Delta^{*}(x)} \ln \operatorname{det}\left[\not \partial+\left(\Sigma(x)-i \gamma^{5} \Pi(x)\right)\right]
$$

$H=-i \gamma^{5} \frac{d}{d x}+\gamma^{0} \Sigma(x)+i \gamma^{1} \Pi(x)=\left(\begin{array}{cc}-i \frac{d}{d x} & \Delta(x) \\ \Delta^{*}(x) & i \frac{d}{d x}\end{array}\right)$

spectral function $\quad \rho(E)=\frac{1}{\pi} \operatorname{Im} \int d x \operatorname{tr} R(x ; E+i \epsilon)$

two views of gap equation:

$$
\begin{aligned}
& \ln \operatorname{det}[\ldots]=-\frac{1}{\beta} \int d E \rho(E) \ln \left(1+e^{-\beta(E-\mu)}\right) \\
& \Delta(x)=-N g^{2} \operatorname{Tr}_{D, E}\left[\gamma^{0}\left(1+\gamma^{5}\right) R(x ; E)\right]
\end{aligned}
$$


gap equation

\section{NLSE}

$$
\frac{\Delta(x)}{g^{2} N}=\frac{\delta}{\delta \Delta^{*}(x)} \ln \operatorname{det}\left[\not \partial+\left(\Sigma(x)-i \gamma^{5} \Pi(x)\right)\right]
$$

$\downarrow$ ansatz, from gap equation

$$
\begin{array}{r}
R(x ; E)=\mathcal{N}(E)\left(\begin{array}{cc}
a(E)+|\Delta(x)|^{2} & b(E) \Delta(x)-i \Delta^{\prime}(x) \\
b(E) \Delta^{*}(x)+i \Delta^{\prime *}(x) & a(E)+|\Delta(x)|^{2}
\end{array}\right) \\
\forall \quad \text { Eilenberger equation } \\
\Delta^{\prime \prime}-2|\Delta|^{2} \Delta+i(b-2 E) \Delta^{\prime}-2(a-E b) \Delta=0
\end{array}
$$

NLSE : exactly soluble; also for exact spectral function 

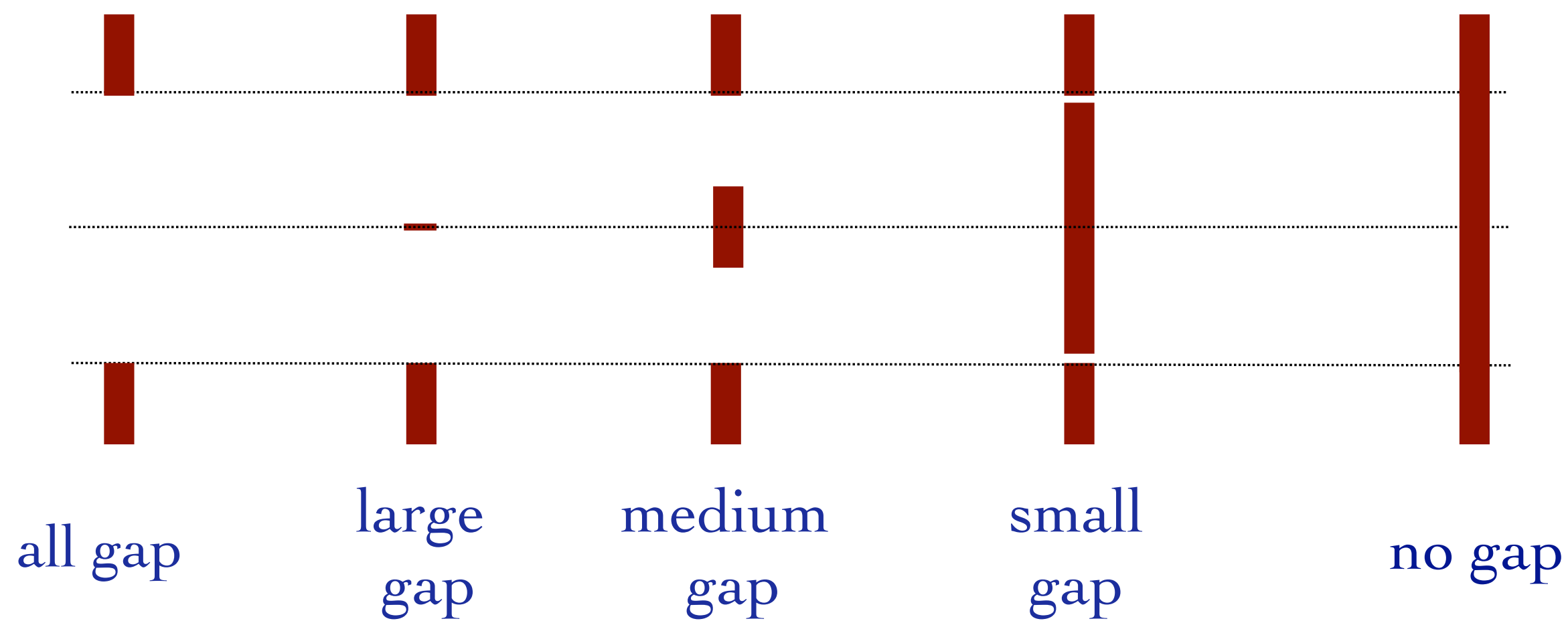

\section{crossing the boundaries ...}

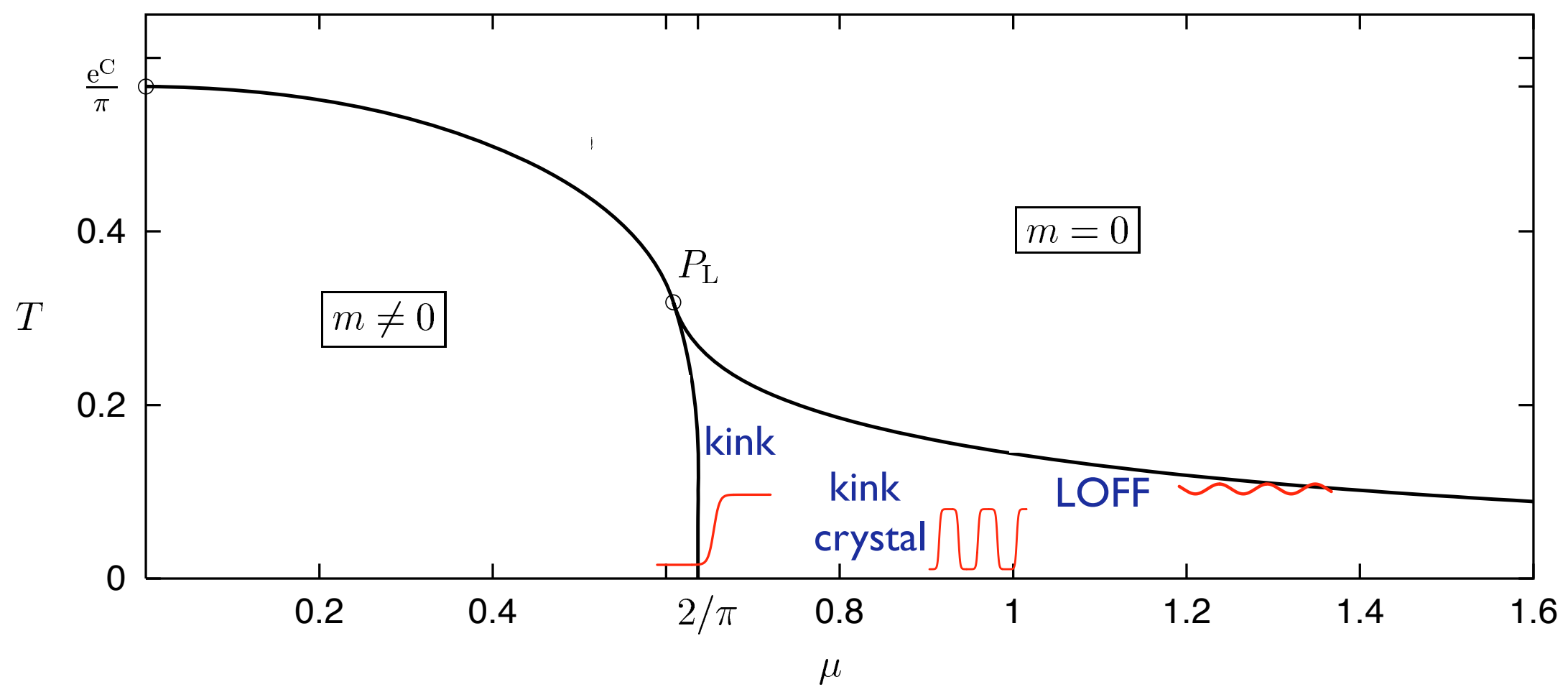


something completely different ... 


\section{Duality and energy-reflection symmetry}

Quasi-exactly-soluble QM models M. Shifman, ITEP lectures

$$
\mathrm{H}=\text { polynomial in } \mathrm{sl}(2, \mathrm{R}) \text { generators }
$$

portion of the spectrum known algebraically

energy-reflection symmetry $\quad$ M. Shifman \& A. Turbiner, 1998

periodic QES systems : energy-reflection duality

GD \& M. Shifman, 2002 
Lamé potential: $V(x)=J(J+1) \nu \operatorname{sn}^{2}(x ; \nu)-\frac{1}{2} J(J+1)$

QES: J bands; edges determined algebraically

$$
\begin{aligned}
& H=J_{x}^{2}+\nu J_{y}^{2}-\frac{1}{2} J(J+1) \mathbf{1} \\
& \nu=0.1 \quad \nu=0.9
\end{aligned}
$$

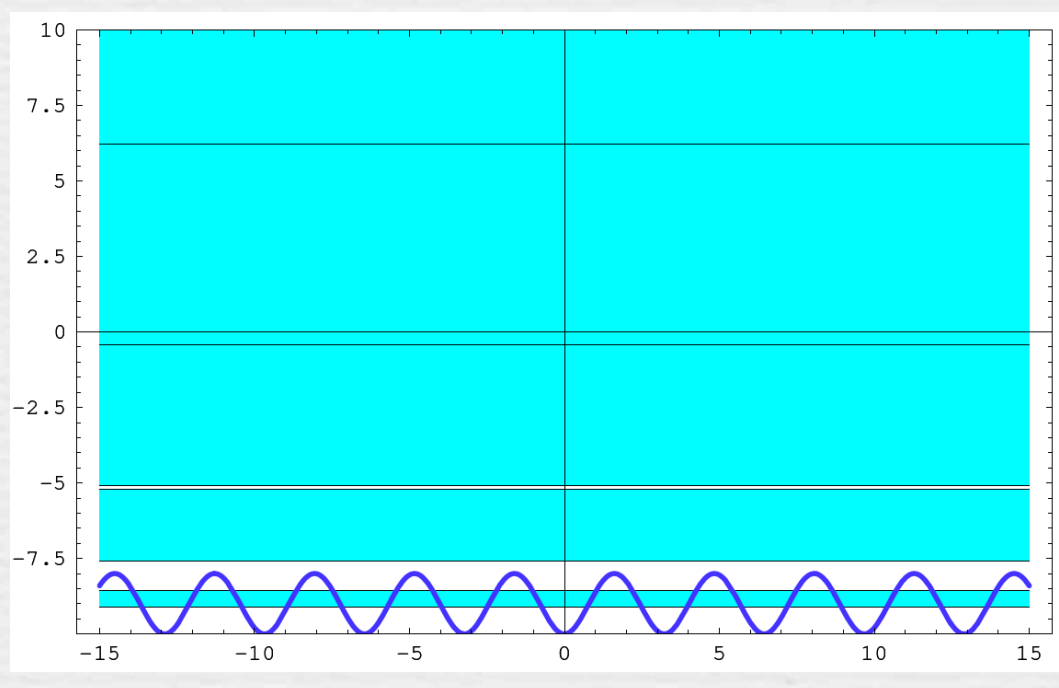

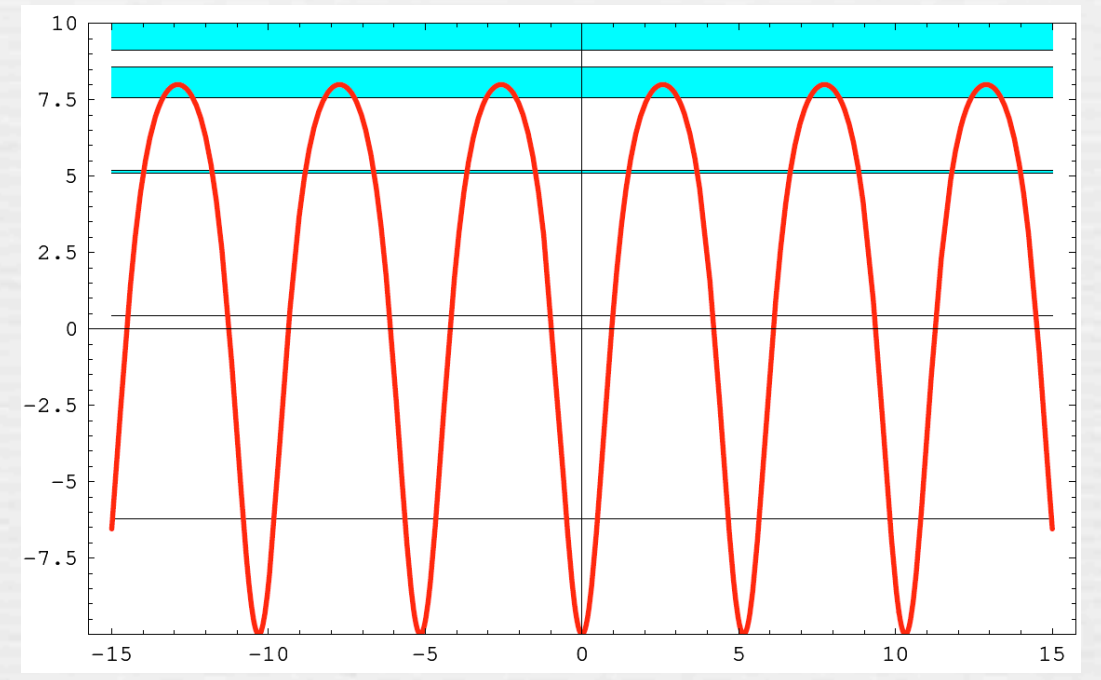

dual potentials

strong coupling <--> weak coupling 
$E$
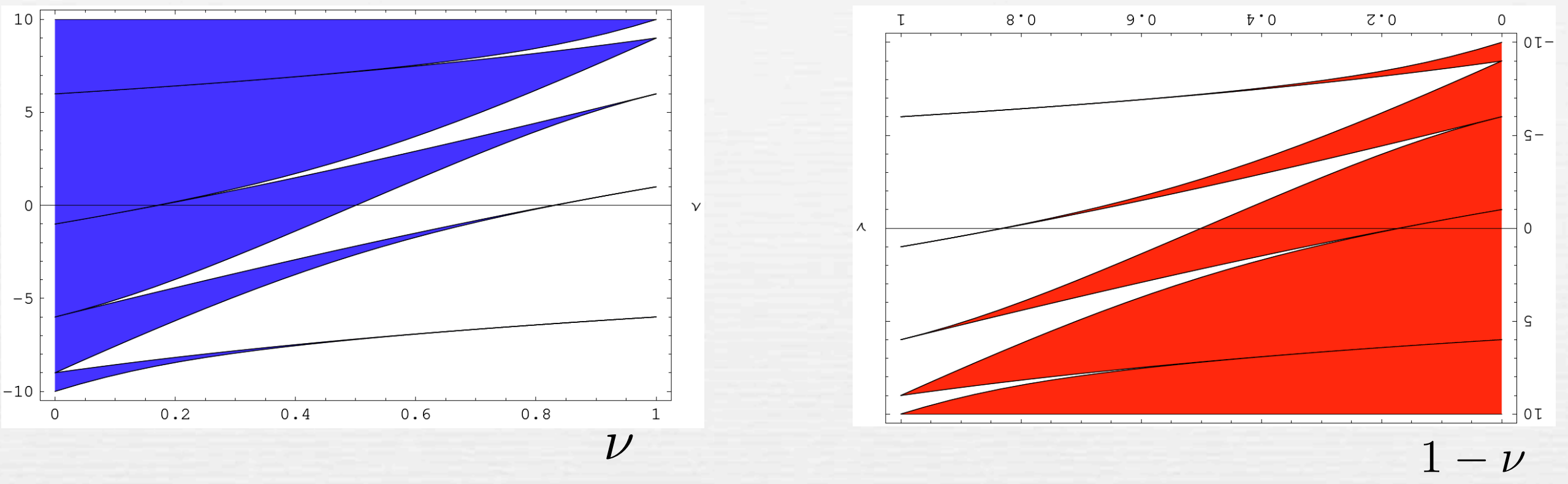

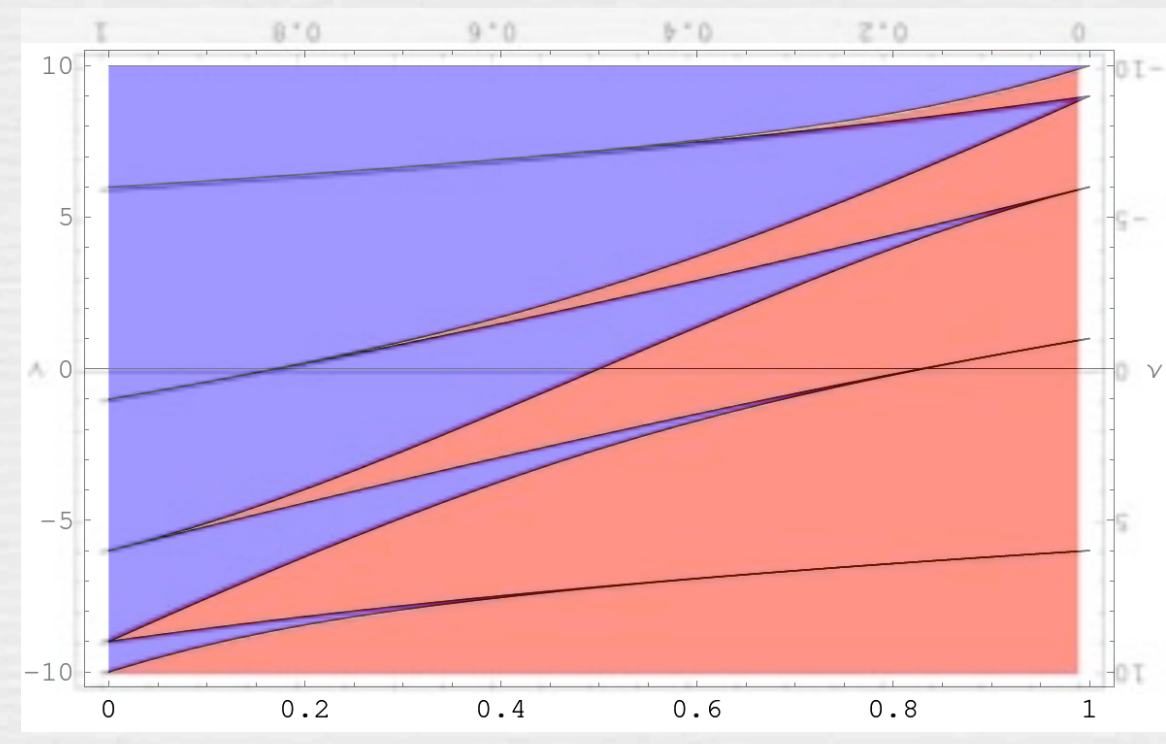




$$
V(x)=J(J+1) \nu \operatorname{sn}^{2}(x ; \nu)-\frac{1}{2} J(J+1)
$$

Мแแแแแบ

गाIIIIIIIIIII

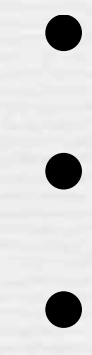

$$
\mathrm{WKB}: E_{\mathrm{top}} \sim \frac{J(J+1)}{2}\left(1-\frac{2 \sqrt{1-\nu}}{\sqrt{J(J+1)}}+\frac{2-\nu}{2 J(J+1)}+\ldots\right)
$$$$
\text { pert. th: } \Delta E_{\text {top }} \sim \frac{8 J \Gamma(J+1 / 2)}{4^{J} \sqrt{\pi} \Gamma(J)}(\nu)^{J}
$$

$$
E[\nu] \leftrightarrow-E[1-\nu]
$$

perturbative/nonperturbative duality

भापापापापा

pert. th: $E_{\mathrm{bottom}} \sim-\frac{J(J+1)}{2}\left(1-\frac{2 \sqrt{\nu}}{\sqrt{J(J+1)}}+\frac{1+\nu}{2 J(J+1)}+\ldots\right)$

$$
\text { instanton : } \Delta E_{\mathrm{bottom}} \sim \frac{8 J \Gamma(J+1 / 2)}{4^{J} \sqrt{\pi} \Gamma(J)}(1-\nu)^{J}
$$




\section{back to Gross-Neveu ...}


Energy-reflection symmetry in the $\mathrm{GN}_{2}$ phase diagram kink crystal condensate: $\Sigma(x)=m \nu \frac{\operatorname{sn}(m x ; \nu) \operatorname{cn}(m x ; \nu)}{\operatorname{dn}(m x ; \nu)}$

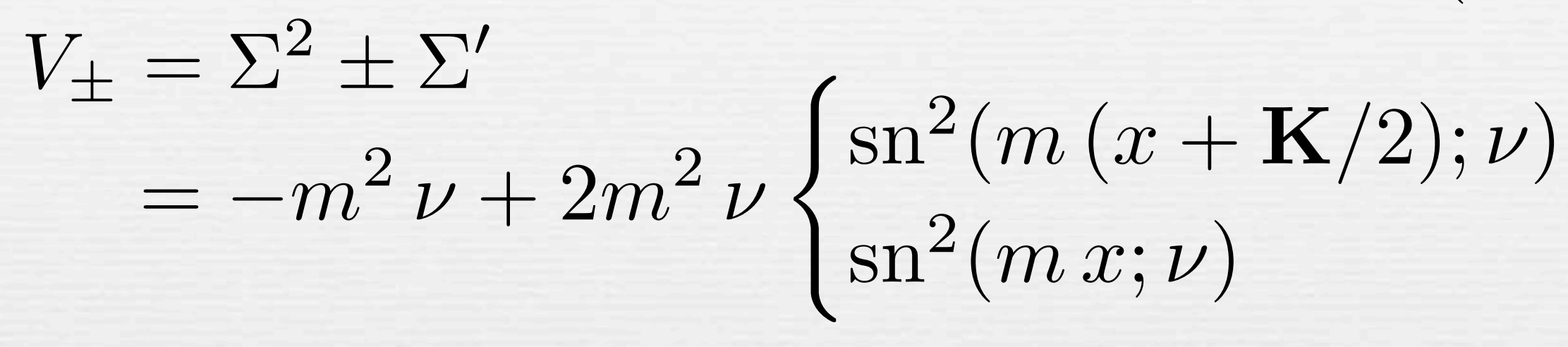

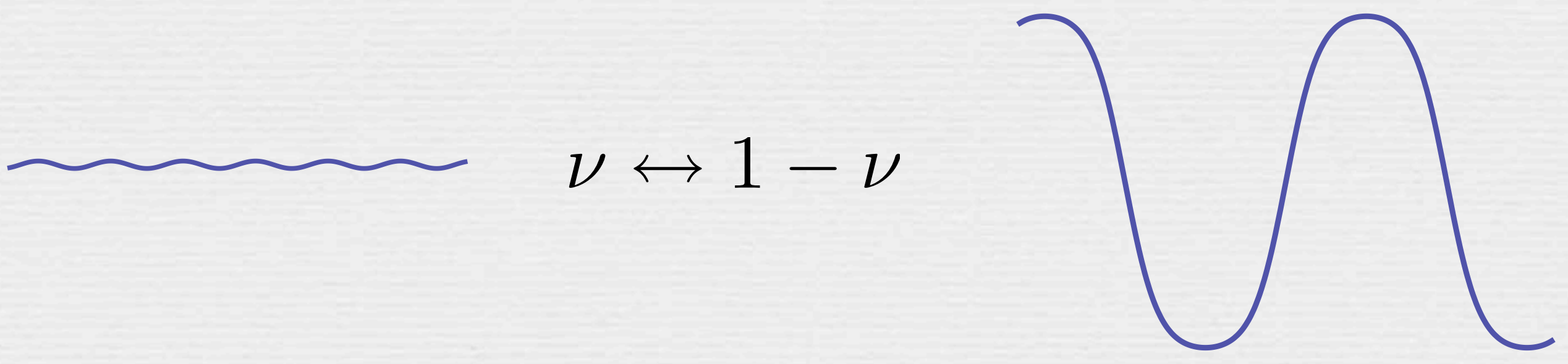

dense $\leftrightarrow$ dilute 


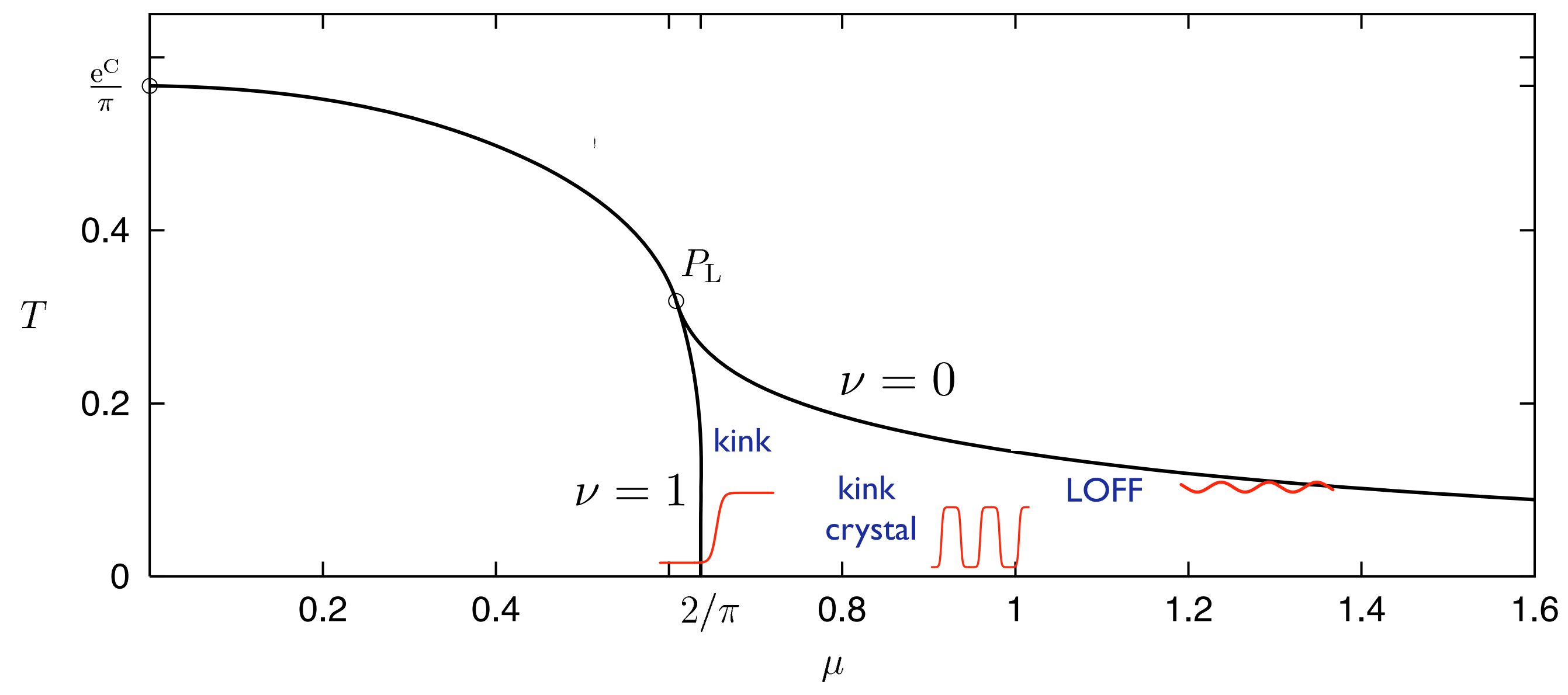

GN phase diagram: dense $<->>$ dilute duality 
Ginzburg-Landau expansion

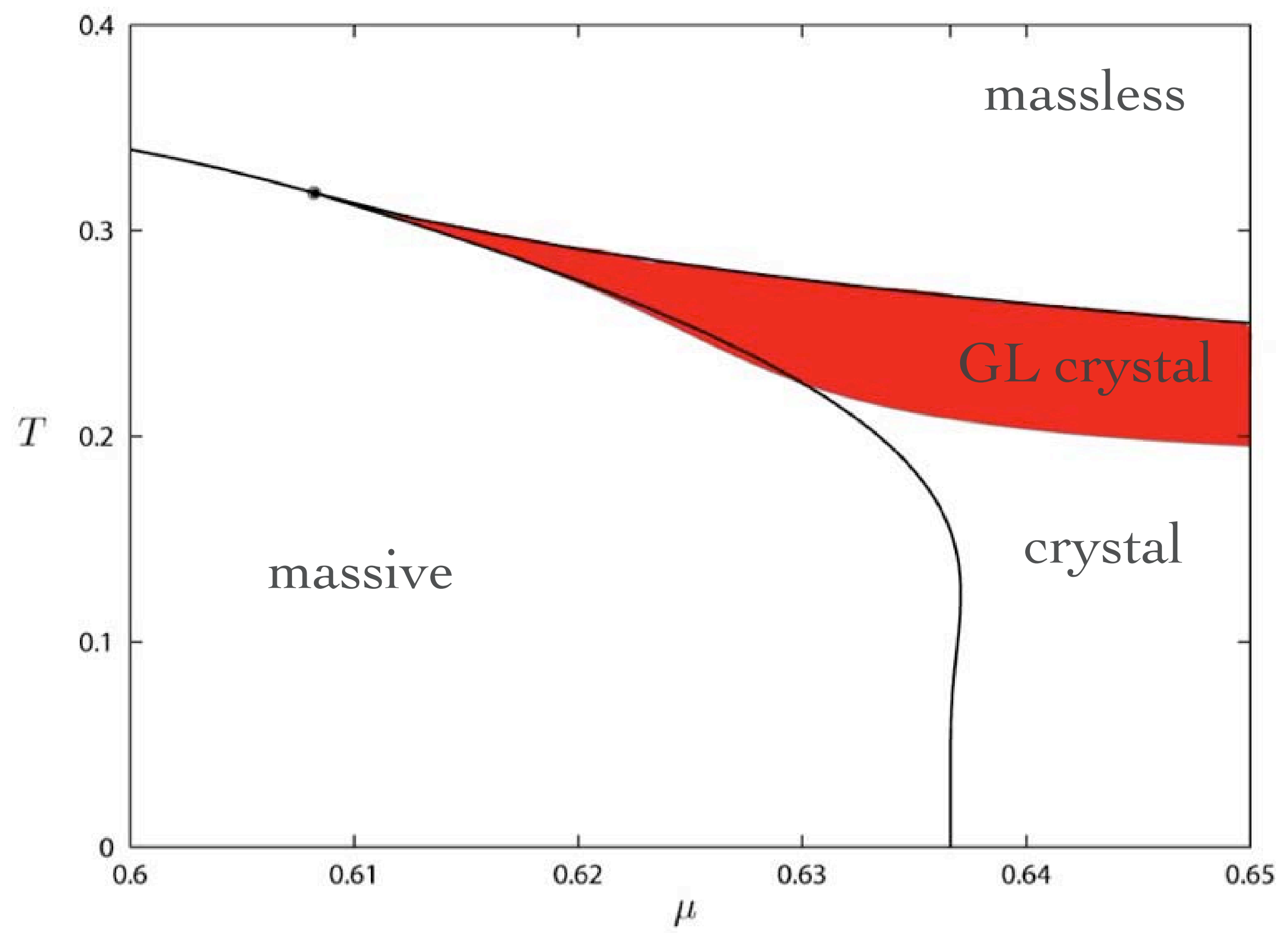


gap equation: all-orders Ginzburg-Landau expansion

$$
\begin{aligned}
\mathcal{L}_{\mathrm{GL}}= & c_{0}+c_{2}|\Delta|^{2}+c_{3} \operatorname{Im}\left[\Delta\left(\Delta^{\prime}\right)^{*}\right]+c_{4}\left[|\Delta|^{4}+\left|\Delta^{\prime}\right|^{2}\right] \\
& +c_{5} \operatorname{Im}\left[\left(\Delta^{\prime \prime}-3|\Delta|^{2} \Delta\right)\left(\Delta^{\prime}\right)^{*}\right] \\
& +c_{6}\left[2|\Delta|^{6}+8|\Delta|^{2}\left|\Delta^{\prime}\right|^{2}+2 \operatorname{Re}\left(\left(\Delta^{\prime}\right)^{2}\left(\Delta^{*}\right)^{2}\right)+\left|\Delta^{\prime \prime}\right|^{2}\right]+\ldots \\
= & \sum_{n} c_{n}(T, \mu) a_{n}(x)
\end{aligned}
$$

NLSE $\rightarrow$ entire hierarchy satisfied

$$
\left[a_{n}(x)\right]_{N L S E}=\alpha_{n}|\Delta(x)|^{2}+\beta_{n}
$$

$\mathrm{GN}_{2}: \mathrm{mKdV}$ hierarchy

$\mathrm{NJL}_{2}$ : AKNS hierarchy 


\section{Conclusions}

- there is a lot of symmetry in the GN phase diagram

- integrable hierachies: $\mathrm{GN}_{2}=\mathrm{mKdV} ; \mathrm{NJL}_{2}=\mathrm{AKNS}$

- thermodynamics: crystalline phases

- energy/reflection symmetry = dense/dilute duality 
Happy Birthday Misha! 


\section{$\mathrm{NJL}_{2}$ phase diagram}

Schön/Thies, 2000

$$
\begin{aligned}
& \text { "chiral spiral" } \\
& \Delta=A e^{2 i \mu x}
\end{aligned}
$$

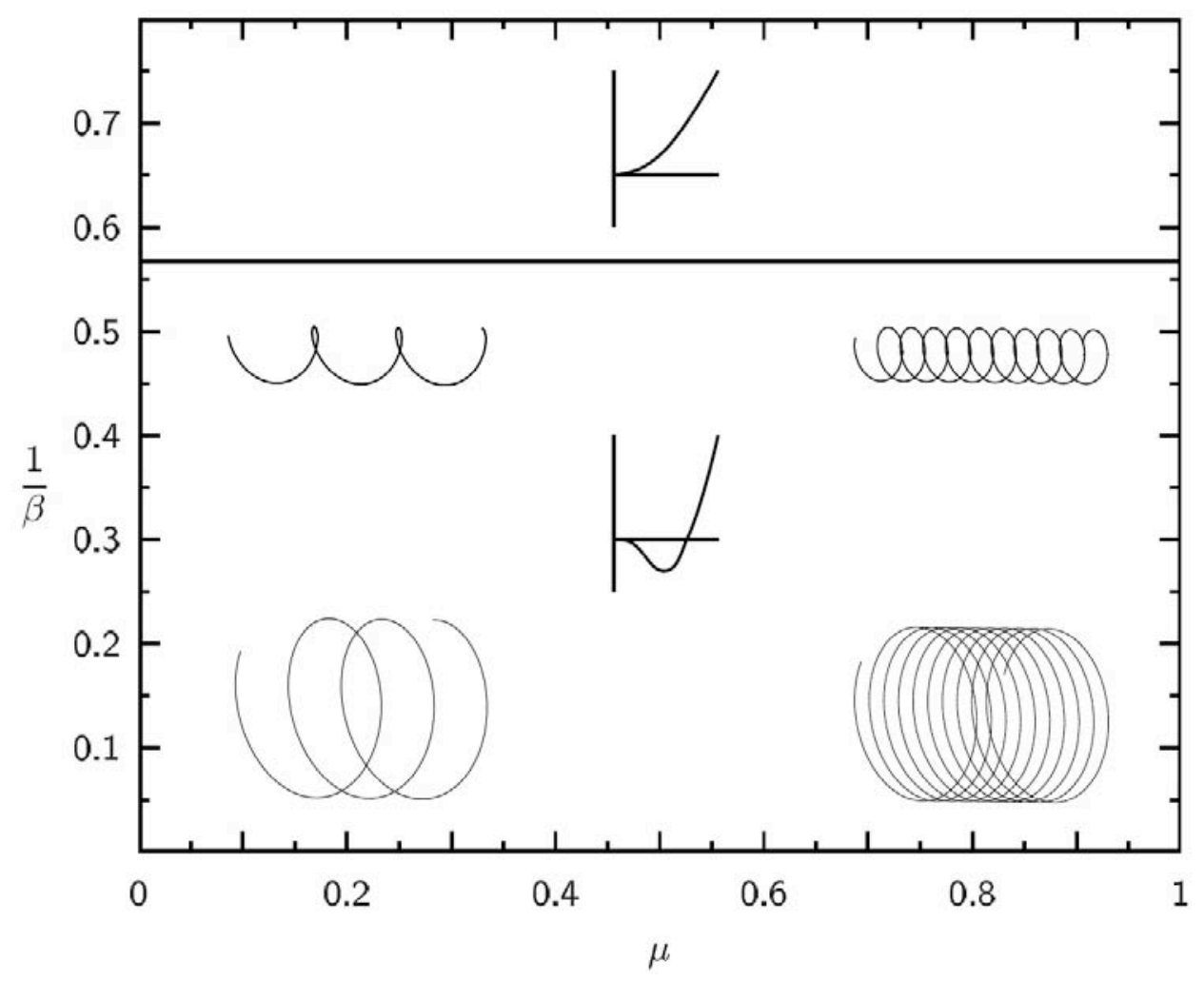

Basar,GD,Thies, 2009: $\quad$ twisted kink crystal --> chiral spiral 